\title{
Screw Cutting Vertebrae After Short-Segment Fixation of Pedicle Screw Combined with Kyphoplasty for Kummell Disease $₫$ A Case Report and Literature Review
}

\section{Xianfu Han}

Gansu University of Traditional Chinese Medicine

Xingwen Xie ( $D$ 827975272@qq.com)

Northwest Minzu University College of Medicine https://orcid.org/0000-0002-7118-5431

\section{Dengyan Bai}

Northwest Minzu University College of Medicine

Lijun Chai

Northwest Minzu University College of Medicine

Juxian Ding

Northwest Minzu University College of Medicine

\section{Technical note}

Keywords: Kummell disease, kyphoplasty, screw cutting vertebra, short-segment fixation of pedicle screw

Posted Date: November 15th, 2021

DOI: https://doi.org/10.21203/rs.3.rs-1039160/v1

License: (c) (1) This work is licensed under a Creative Commons Attribution 4.0 International License.

Read Full License 


\section{Abstract}

Introduction: Kummell disease is also known as vertebral fracture nonunion, delayed vertebral collapse and vertebral ischemic necrosis. It often occurs in a delayed manner after spinal injury, and is a complex and rare spinal disease. This study, aimed to report a case of stage 3 Kummell disease, diagnosed using magnetic resonance imaging (MRI) of the lumbar spine.

Methods: The subject underwent a short-segment fixation of the pedicle screw combined with kyphoplasty.

Results: The patient was satisfied with the relief of back pain after the surgery, but the surgery was found to cause screw cutting vertebrae in the second lumbar vertebra (L2).

Conclusion: Short-segment fixation of the pedicle screw combined with kyphoplasty can reconstruct spinal stability, maintain stability of bone cement mass in vertebrae, correct kyphosis deformity, and relieve pain. However, Kummell disease in elderly patients is always accompanied by severe osteoporosis. The common fixation of the pedicle screw is prone to cause screw cutting, loosening, and pulling out. Therefore, we advocate using cement-augmented pedicle screws.

\section{Introduction}

Kummell disease was discovered and proposed as vertebral fracture nonunion and vertebral ischemic necrosis by Dr. Hermann Kummell in 1891. It is a special type of osteoporotic vertebral compression fracture, common in elderly individuals. Following a short asymptomatic period after spinal injury, patients often develop recurrent back pain, progressive vertebral compression collapse and kyphosis.

The pathogenesis of Kummell disease is mainly vertebral ischemic necrosis and pseudarthrosis formation based on osteoporosis [1, 2]. It is also related to other factors that may affect vertebral bone mineral density and blood supply, such as long-term alcoholism, long-term hormone use, bone metabolism diseases, and so forth [3].

The most widely used classification method for Kummell disease was proposed by Kung et al. At present. Stage 1 has a body height loss of $<20 \%$ and no adjacent degenerative disk disease (DDD). Stage 2 has a body height loss of $>20 \%$ and is usually associated with adjacent DDD; dynamic active fault is the main feature of this stage. Stage 3 has posterior breakage and cord compression [4].

Surgical treatment should be carried out in time to avoid the recurrence of back pain. Many scholars believe that percutaneous kyphoplasty (PKP) or percutaneous vertebroplasty (PVP) is an appropriate treatment for stage 1 or 2 Kummell disease. Also, the vertebral quality is acceptable, the collapse and compression are incomplete, the posterior cortex of the centrum is intact, and no obvious neurological symptoms are found, but the two treatments have a high risk of bone cement leakage [5]. 
For patients with stage 3 Kummell disease, posterior decompression with osteotomy, anterior decompression with fusion or combine anterior with posterior surgery can be used. However, the procedure for the anterior approach is long and may damage the internal organs. The posterior decompression osteotomy is traumatic, with excessive bleeding. Further, the elderly patients often have chronic diseases and cannot tolerate the long-term prone operation. No standard surgical treatment methods are available for stage 3 Kummell disease [6].

This study described the short-segment fixation of the pedicle screw combined with kyphoplasty in treating stage 3 Kummell disease. Unfortunately, the surgery caused screw cutting vertebrae.

\section{Case Presentation And Surgical Condition}

A 79-year-old woman with severe osteoporosis complained of her back and right lower limb pain for 1 week after a sprain at home. She had severe back pain, limited activities, difficulty in turning over, weakness of both lower limbs when standing, and no relief after bed rest. The patient described pain in her right lower limb and weakness in both legs. Two years ago, right hip arthroplasty was performed. The patient had hypertension for 10 years and a history of diabetes.

The physical examination revealed thoracolumbar kyphosis deformity, tenderness around the the first lumbar vertebra(L1)-the fourth lumbar vertebra, and an obvious percussion pain in the back. The muscle strength of both lower limbs was grade 4, the skin sensation of both lower limbs was slightly decreased, the right Achilles tendon reflex was weakened, the bilateral Babinsky sign was negative, and no incontinence was observed.

After hospitalization, we considered that the injury lesion was in the lumbar area based on medical history. We performed lumbar x-ray, computed tomography (CT), and MRI. The x-ray showed that L1 had an obvious intravertebral vacuum cleft (IVC) (Fig. 1). Obvious IVC was detected on CT (Fig. 2). The MRI cross-section showed the vacuum sign in the centrum. On sagittal T1-weighted MRI, the signal of IVC and posterior cortex rupture in L1 decreased, and the spinal cord was compressed continuously. Sagittal T2weighted MRI showed a signal of IVC and posterior cortex rupture in L1 (Fig. 3).

The surgery was performed in the prone position and with general anesthesia. A good posterior exposure of the spine was given using the Wiltse surgical approach. Further, the pedicle screw was inserted into the twelfth thoracic vertebra (T12) and L1 under $\mathrm{C}$-arm guidance. The two rods were bent to the required contour, the rods were connected with screws, and the expander was used to expand L1.C-arm fluoroscopy showed that the height of $L 1$ recovered to about one third of the original height. The needle was inserted into the right pedicle of $L 1$ to establish the bone channel, and then the balloon dilator was placed to stretch the L1. The height of L1 was restored to one half of the original height, and then the balloon dilator was withdrawn. After inserting a bone cement working sleeve, the polymethylmethacrylate (PMMA) cement was pushed and injected. Then, the expander expanded and reset L1, the PMMA cement was continuously pushed. After the PMMA cement solidified, the working sleeve was taken out. C-arm fluoroscopy showed that the height of L1 was partially recovered, kyphosis was slightly corrected, bone 
cement dispersion was good and no leakage occurred. However, pedicle screw cutting vertebrae were noted in L2 (Fig. 4). After the bleeding stopped, the wound was thoroughly rinsed with sterile normal saline and closed layer by layer. The operation time was $70 \mathrm{~min}$, and the total blood loss was $300 \mathrm{~mL}$.

After the surgery, the patient was given antibiotics and anticoagulant therapies for 3 days, and pain treatment and nutritional nerve treatment for 5 days. She also received regular treatment for hypertension, diabetes, and anti-osteoporosis, and also stayed in bed. The drainage tube was removed after two days when the drainage volume was less than $50 \mathrm{~mL} / 24 \mathrm{~h}$ after the surgery. Six days after the surgery, the patient's family asked for discharge from the hospital. The patient was told to stay in bed for 4 weeks. After 4 weeks, she was discharged with customized orthosis, and the orthosis was used for at least 3 months.

A visual analogue scale (VAS) was used to evaluate the pain in the postoperative period. The VAS score before and 3 days after the surgery was 9 and 2 respectively, which was significantly improved. No change in muscle strength and skin sensation of lower limbs was observed after the surgery. The x-ray of the lumbar spine after the surgery revealed the screw cutting vertebrae in L2. Also, the height of L1 was partially recovered, the kyphosis was slightly corrected, and bone cement dispersion was good (Fig. 5).

\section{Discussion}

This study reported the treatment of Kummell disease with short-segment fixation of the pedicle screw combined with kyphoplasty. Unfortunately, the surgery led to screw cutting vertebrae. The treatment could reconstruct spinal stability, maintain stability of bone cement mass in vertebrae, correct kyphosis deformity, and relieve pain, but complications such as bone cement leakage and pulmonary embolism occurred. The fusion rate in Kummell disease is worrying. Consequently, simple bone particle fusion takes a long time and is prone to bone particle displacement, resulting in poor curative effect. Bone cement, as a common therapeutic agent for treating spinal compression fractures, has reliable curative effect, short solidification time and rapid recovery. It is more suitable for this kind of surgery [5]. However, while treating stage 3 Kummell disease with simple kyphoplasty, the dislocation of bone cement mass occurred 1 month after the surgery and the injured vertebrae lost its effective support, resulting in the aggravation of kyphosis and partial paralysis of the patient [7].

Kummell disease is characterized by advanced age, high surgical risk, osteoporosis and low fusion rate. Some patients do not tolerate this kind of surgery. Therefore, some scholars used internal fixation combined with augmented bone cement to achieve long-term spinal stability. On the basis of posterior internal fixation, bone cement was injected into the injured vertebrae to enhance the stability of the spine. After internal fixation, the injured vertebrae were reduced to form a local empty shell. If only posterior pedicle screw fixation was adopted at this time, pedicle screw fracture might occur after a long time of local stress. Therefore, internal fixation combined with augmented bone cement was a better treatment [8, 9]. PVP or PKP could be used to strengthen the injured vertebrae. Both of them provided similar ain relief, but PKP is superior to PVP in restoring vertebral height and reducing cement leakage [10]. 
Gan et al reported that using short-segment percutaneous pedicle screw fixation combined with vertebroplasty in treating stage 3 Kummell disease could effectively restore the height of the injured vertebrae, correct kyphosis, reduce trauma, prevent the injured vertebrae from collapsing again, and effectively improve clinical symptoms. No loosening of pedicle screws was found in the follow-up [11]. Lou et al reported that long-segment percutaneous pedicle screw fixation combined with vertebroplasty was also an effective minimally invasive treatment for Kummell disease with scoliosis and intraspinal instability; However, a certain number of cases also experienced pedicle screw loosening after the surgery [12]. Long-segment pedicle screw fixation provided satisfactory orthopedic effect and strong fixation, but it affected the spinal function and was associated with immense trauma, excessive bleeding, and high cost. Short-segment fixation involves lesser trauma and lesser blood loss, but it has some shortcomings such as orthopedic loss and internal fixation failure. At present, no standard exists for long-segment or short-segment fixation. Both of them have advantages and disadvantages.

In the preoperative discussion of this case, we determined two surgical schemes. Scheme 1, after T12 and L1 of pedicle screw internal fixation, could not expand and restore the height of L1 or the recovery of the height of L1 was not satisfactory. Therefore, we planned to use osteotomy and orthopedic surgery. Scheme 2, after T12 and L1 of pedicle screw internal fixation, could expand and restore the height of L1. We used internal fixation combined with kyphoplasty. During the surgery, we inserted T12 and L1 pedicle screws, connected the screw and rods, expanded and restored the height of L1. We found that the height of $L 1$ was partially recovered, and we decided to use kyphoplasty. During the injection of PMMA cement, the expander expanded and restored the height of L1. It was found that the surgery caused screw cutting L2. The screw of T12 was placed high and close to the upper endplate cortex, and did not cause cutting vertebrae. If the adjacent vertebrae of the injured vertebrae are filled with bone cement and the pedicle screw is inserted, the safety of restoration may be higher after the bone cement is cooled and solidified and then expanded and restored, but the operation time may be longer. Elderly patients often have chronic diseases and high operation risk, and hence cannot tolerate long-term prone surgery. Therefore, we did not replace the common pedicle screw with bone cement screw during the surgery.

In this case, the operation caused screw cutting vertebrae mainly because the elderly patient had severe osteoporosis, serious bone loss, and sparse bone trabeculae. Also, the connection of the bone-screw interface was unstable, the force of distraction reset for the pedicle screw was excessive. If the postoperative activity is too large or early, the standard anti-osteoporosis treatment is not continued, as it may increase the degree of screw cutting, affect the strength and stability of pedicle screw fixation, and damage the adjacent tissue of vertebrae [13].

Spinal surgeons have to encounter osteoporotic spinal diseases more frequently with the increase in the global elderly population and the influence of other risk factors. Osteoporotic spinal diseases can cause many problems in the treatment, especially when instruments are used, resulting in screw cutting and damage to vertebrae, or even loosening, pulling out, or fracture. Scholars from various countries have made various improvements in screw design and surgical techniques to improve the fixation strength and stability of pedicle screws in patients with osteoporosis. The screw design includes increasing the 
strength of the screw-bone interface with bone cement and changing the screw diameter, length, thread design, conical design of screw inner diameter, and expandable screw [14-17].The improvement in surgical technology mainly includes the advancement in screw placement technology, cortical bone trajectory, superior cortical trajectory, cross trajectory technology, double cortical fixation, and double screw fixation technology $[18,19]$.

In theory, cement, as a bone adhesive, can firmly connect the implant material and bone to enhance its firmness. In recent years, many scholars and doctors carried out biomechanical and clinical research on the use of augmented-cement pedicle screws; They found that these screws could improve the stability and reliability of internal fixation in patients with osteoporosis [20-22]. When we used the fixation of cement-augmented pedicle screw, the bone-screw interface was transformed into the bone-cementscrew interface, and the screw and cement were closely combined so as to overcome the defect that the bone-screw interface was not firmly connected, resulting in the pulling out of the screw. The screw was

fixed after the bone cement was injected into the screw path. The screw was wrapped with bone cement and placed in contact with the bone. The contact area increased and the interaction force dispersed, resulting in reducing the destructive cutting force of the screw acting on the vertebrae [23]. Ji et al used cement-augmented pedicle screws that were inserted into fresh cadaveric thoracolumbar spine specimens; biomechanical tests were performed on an electronic universal mechanical testing machine. In the experiment, the force of the upward screw was measured along the longitudinal axis of the human body. The withstand load threshold was the force when vertebrae were damaged and screws were pulled out. Then, the obtained data were analyzed. He found that bone cement had a significant strengthening effect on the tolerance of screw cutting and damage to the vertebrae. The withstand load threshold of cement-augmented pedicle screws was $(1198 \pm 122) \mathrm{N}$, and that of pedicle screws was $(805 \pm 64) \mathrm{N}$. Also, cement-augmented pedicle screw internal fixation had a stronger mechanical effect and a specific role in preventing screw cutting and damage to the vertebrae, which improved the curative effect of internal fixation in patients with osteoporosis [24]. At present, the use of cement-augmented pedicle screw in treating Kummell disease is common, with high fixation strength and stability, and satisfactory results [25-26]. Most patients with Kummell disease always have severe osteoporosis. Therefore, we advocate using cement-augmented pedicle screw fixation so as to prevent screw cutting and loosening of internal fixation. Also, postoperative anti-osteoporosis measures should be taken.

\section{Conclusion}

Short-segment fixation of pedicle screw combined with kyphoplasty can reconstruct spinal stability, maintain the stability of bone cement mass in vertebrae, correct kyphosis deformity and relieve pain. However, Kummell disease is often accompanied by severe osteoporosis in elderly patients. The common fixation of the pedicle screw is prone to cause screw cutting vertebrae. Therefore, we advocate using cement-augmented pedicle screws. However, this was only a case report, and hence more cases should be examined to confirm the conclusion. 


\section{Abbreviations}

DDD: degenerative disk disease; IVC: intravertebral vacuum cleft; L1: the first lumbar vertebra; L2: the second lumbar vertebra; MRI: magnetic resonance imaging; PKP: percutaneous kyphoplasty; PMMA: polymethylmethacrylate; PVP: percutaneous vertebroplasty; T12: the twelfth thoracic vertebra; VAS: visual analogue scale

\section{Declarations}

\section{Acknowledgments}

The authors would like to acknowledge and thank the individual patient involved who agreed to the publication of this article.

\section{Authors' contributions}

XF.H was responsible for the data collation and primary manuscript generation. XW.X was the senior author who performed the surgery and contributed to study design. DY.B was responsible for manuscript editing. LJ.C was responsible for the data collation. JX.D was responsible for language proofreading. The author(s) read and approved the final manuscript.

\section{Funding}

This work was funded by the National Natural Science Foundation of China (grant no.: 81860864 , Xingwen Xie)

\section{Availability of data and materials}

Data sharing is not applicable to this article as no datasets were generatedor analyzed during the current study.

\section{Ethics approval and consent to participate}

The patient signed an informed consent form. They agreed to provide permission for the de-identified images as well as details of the case to be used for publication.

\section{Consent for publication}

Consent for publication was obtained from the participant. 


\section{Competing interests}

All authors declare no competing interests.

\section{Author details}

${ }^{1}$ Clinical Medical College of Traditional Chinese Medicine, Gansu University of Chinese Medicine, Lanzhou 730000, Gansu, China.

${ }^{2}$ Department of Orthopedics, Gansu Second Provincial People's Hospital, Lanzhou 730020, Gansu, China.

${ }^{3}$ Department of Orthopedics, Affiliated Hospital of Northwest Minzu University, Lanzhou 730020, Gansu, China.

\section{References}

1. Kim YC, Kim YH, Ha KY. Pathomechanism of intravertebral clefts in osteoporotic compression fractures of the spine. Spine J. 2014; 14:659-66.

2. Kong LD, Wang P, Wang LF, Shen Y, Shang ZK, Meng LC. Comparison of vertebroplasty and kyphoplasty in the treatment of osteoporotic vertebral compression fractures with intravertebral clefts. Eur J Orthop Surg Traumatol.2014; 24: S201-208.

3. Matzaroglou C, Georgiou CS, Assimakopoulos K, Giannakenas C, Karageorgos A, Saridis A, et al. Kümmell's disease: pathophysiology, diagnosis, treatment and the roleof nuclear medicine. Rationale according to our experience. Hell J Nucl Med. 2011; 14:291-9.

4. KungChia L, TakUee W, FuChi K, et al. Staging of Kummell's disease. J Musculoskelet Res. 2004; 8:43-55.

5. Yu H, Li Y, Yao X, Lin J, Pan Y, Zhuang H, et al. Application of percutaneous vertebroplasty and percutaneous kyphoplasty in treating Kümmell's patients with different stages and postural correction status. Expert Rev Med Devices.2020;17:357-364.

6. Liu F, Chen Z, Lou C, Yu W, Zheng L, He D, et al. Anterior reconstruction versus posterior osteotomy in treating Kümmell's disease with neurological deficits: A systematic review. Acta Orthop Traumatol Turc.2018;52:283-288.

7. Zhang C, Wang G, Liu X, Li Y, Sun J. Failed percutaneous kyphoplasty in treatment of stage 3 Kummell disease: A case report and literature review. Medicine (Baltimore).2017; 96: e8895.

8. Wang B, Wang Y, Zhang H, Yan L, Kong L, He S, et al. A novel bone cement screw system combined with vertebroplasty for the treatment of Kummell disease with bone deficiency at the vertebral anterior border: A minimum 3-year follow-up study. Clin Neurol Neurosurg.2021;201:106434.

9. Zhao JP, Sun C, Xiang XQ, Liu ZH, Wu HA, Du SY. Cement augmented pedicle screw combined with vertebroplasty for the treatment of Kümmell's disease with type âđç. Zhong guo Gu 
Shang.2021;34:170-4.

10. Zhang B, Chen G, Yang X, Fan T, Chen Z. Percutaneous Kyphoplasty Versus Percutaneous Vertebroplasty for Neurologically Intact Osteoporotic Kümmell's Disease: A Systematic Review and Meta-Analysis. Global Spine J. 2021; Epub ahead of print.

11. Gan DH, Fang MZ, Xue HP, Tan GQ, Li NH, Li ZC, et al. Clinical Observationsof Kümmell Disease Treatment Through Percutaneous Fixation Combined with Vertebroplasty. Orthop Surg. 2021; 13:1505-1512.

12. Lou C, Yu W, Chen Z, Gao J, Liu F, He D. Short-term outcomes of percutaneous pedicle screw fixation combined with vertebroplasty: A minimally invasive treatment for Kümmell's disease with intravertebral instability. Acta Orthop TraumatolTurc.2020;54:627-633.

13. Amirouche F, Solitro GF, Magnan BP. Stability and Spine Pedicle Screws Fixation Strength-A Comparative Study of Bone Density and Insertion Angle. Spine Deform.2016;4:261-267.

14. Elder BD, Lo SF, Holmes C, et al. The biomechanics of pedicle screw augmentation with cement. Spine J.2015; 15:1432-45.

15. Viezens L, Sellenschloh K, Püschel K, Morlock MM, Lehmann W, Huber G, et al. Impact of Screw Diameter on Pedicle Screw Fatigue Strength-A Biomechanical Evaluation. World Neurosurg. 2021;152: e369-e376.

16. Matsukawa K, Yato Y, Imabayashi H. Impact of Screw Diameter and Length on Pedicle Screw Fixation Strength in Osteoporotic Vertebrae: A Finite Element Analysis. Asian Spine J.2020; Epub ahead of print.

17. Gazzeri R, Panagiotopoulos K, Galarza M, Bolognini A, Callovini G. Minimally invasive spinal fixation in an aging population with osteoporosis: clinical and radiological outcomes and safety of expandable screws versus fenestrated screws augmented with polymethylmethacrylate. Neurosurg Focus.2020; 49: E14.

18. Paik H, Dmitriev AE, Lehman RA Jr, Gaume RE, Ambati DV, Kang DG, et al. The biomechanical effect of pedicle screw hubbing on pullout resistance in the thoracic spine. Spine J. 2012; 12:417-24.

19. Karikari IO, Metz LN. Preventing Pseudoarthrosis and Proximal Junctional Kyphosis: How to Deal with the Osteoporotic Spine. Neurosurg Clin N Am.2018;29:365-374.

20. Weiser L, Sellenschloh K, Püschel K, Morlock MM, Viezens L, Lehmann W, et al. Reduced cement volume does not affect screw stability in augmented pedicle screws. Eur Spine J. 2020; 29:12971303.

21. Kim JH, Ahn DK, Shin WS, Kim MJ, Lee HY, Go YR. Clinical Effects and Complications of Pedicle Screw Augmentation with Bone Cement: Comparison of Fenestrated Screw Augmentation and Vertebroplasty Augmentation.Clin Orthop Surg.2020;12:194-199.

22. Xiu J, Bu T, Yan Y, Wu Z, Yin Z, Lei W. Biomechanical study of space frame structure based on bone cement screw. Exp Ther Med.2020; 19:3650-3656.

23. Prokop A, Chmielnicki M. Perkutane Versorgung von instabilen Wirbelfrakturen - OP-Video und Ergebnisse vonüber 300 Fällen [Percutaneous treatment of unstable spine fractures - OP video and 
results from over 300 cases]. ZOrthop Unfall.2014;152:15-7.

24. Ji HY, Huo HJ. Biomechanics Effect of Pedicle Screw with PMMA in Internal Fixation of Spine. Journal of Practical Orthopaedics.2011;17:36-40.

25. Wang Z, Liu Y, Rong Z, Wang C, Liu X, Zhang F, et al. Clinical evaluation of a bone cement-injectable cannulated pedicle screw augmented with polymethylmethacrylate: 128 osteoporotic patients with 42 months of follow-up. Clinics (Sao Paulo).2019;74: e346.

26. Di HX, Liu FY, Yang SD, Wang H, Yang DL, Ding WY. Short-segment fixation with a cement-augmented pedicle screw for Kummell disease: Case report. Medicine (Baltimore).2017; 96: e8617.

\section{Figures}

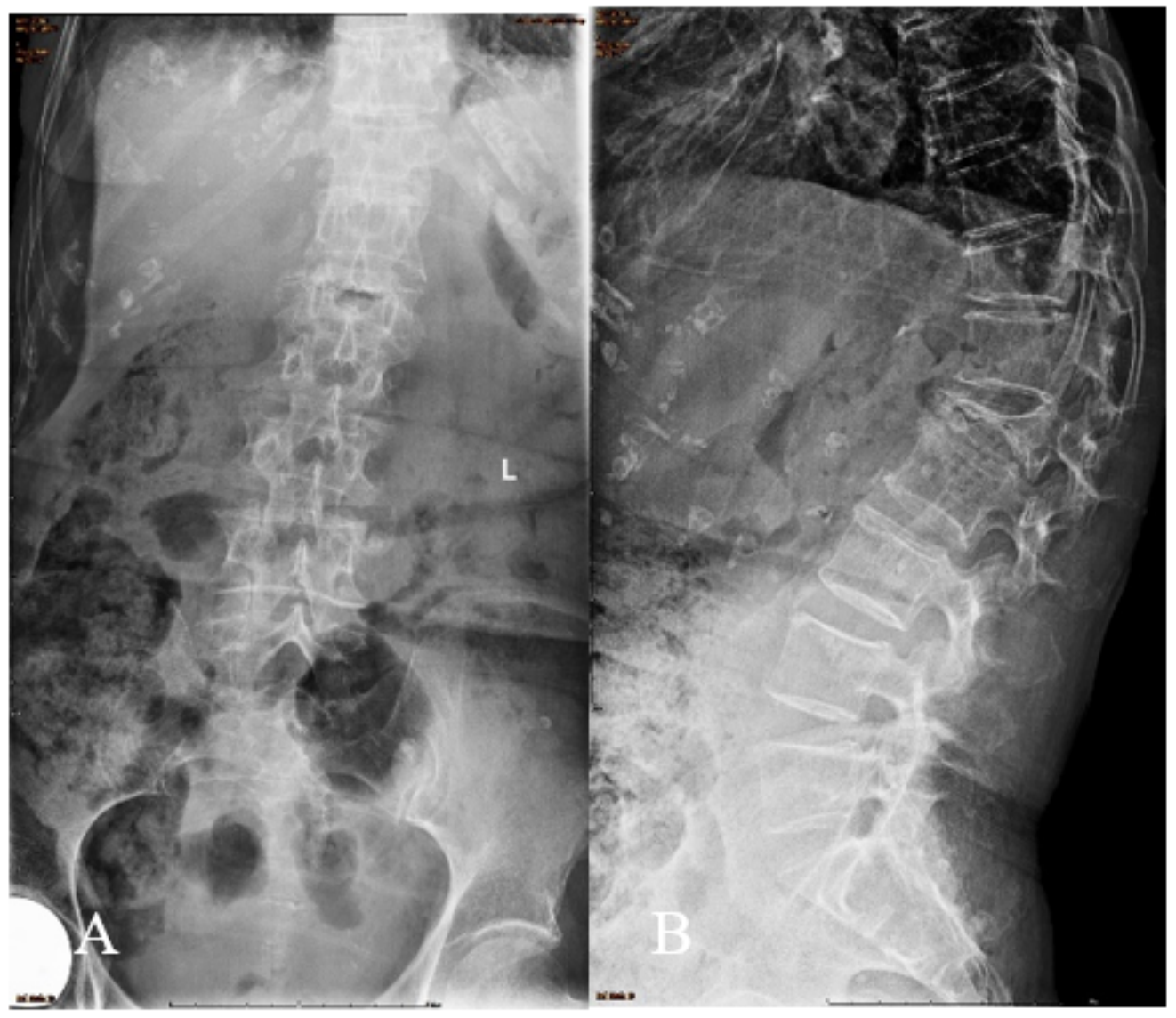

\section{Figure 1}

( $A$ and B) Preoperative positive and lateral $x$-ray. ( $A$ and B) Preoperative positive and lateral $x$-ray show obvious intravertebral vacuum cleft (IVC) of L1. 


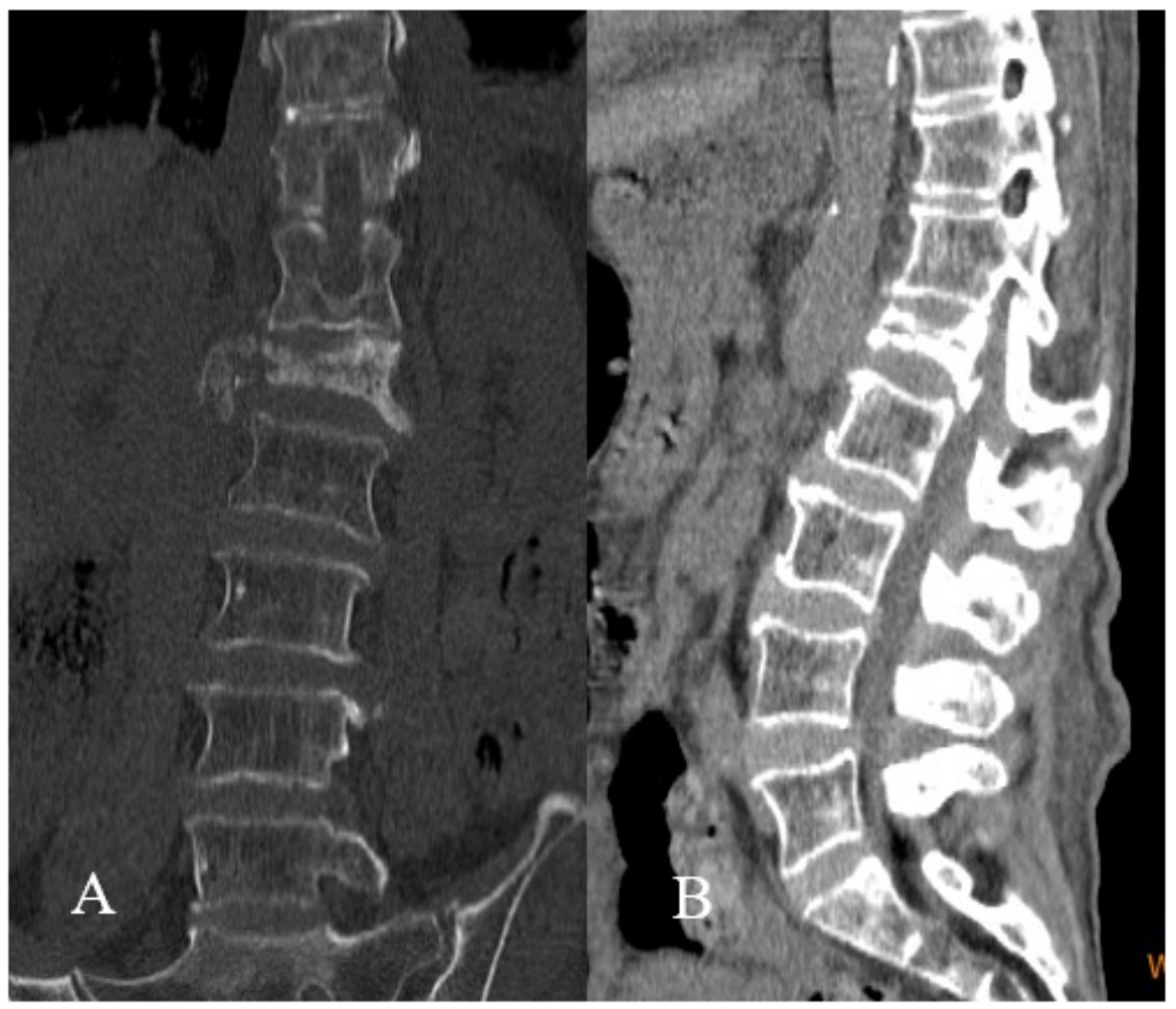

Figure 2

(A and B) Preoperative positive and lateral CT. (A and B) Preoperative positive and lateral CT reveals obvious IVC of L1.

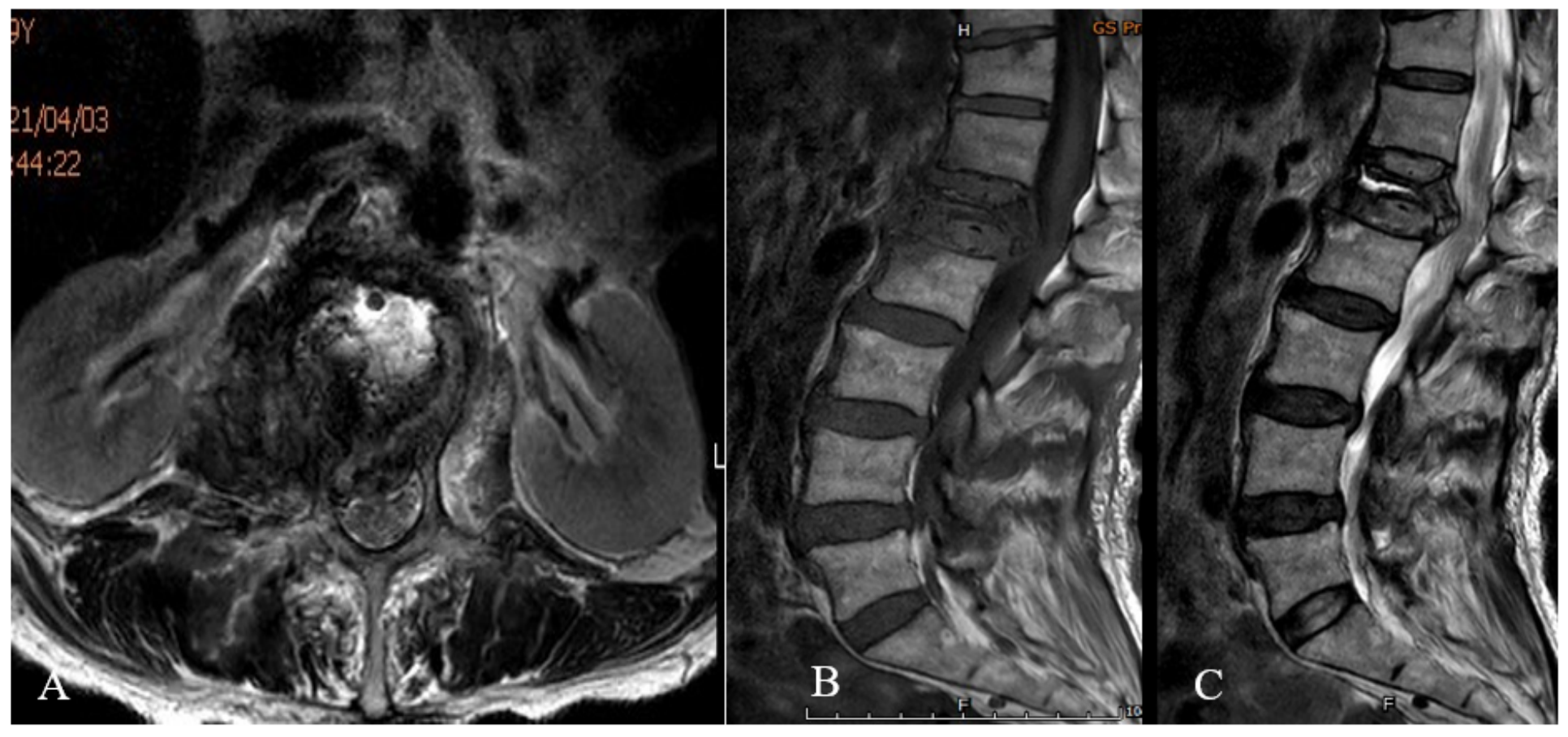

Figure 3 
(A-C) Preoperative MRI. (A-C) Preoperative MRI shows L1 Kummell disease and obvious compression on the spinal cord. Also, have posterior cortex rupture in L1.

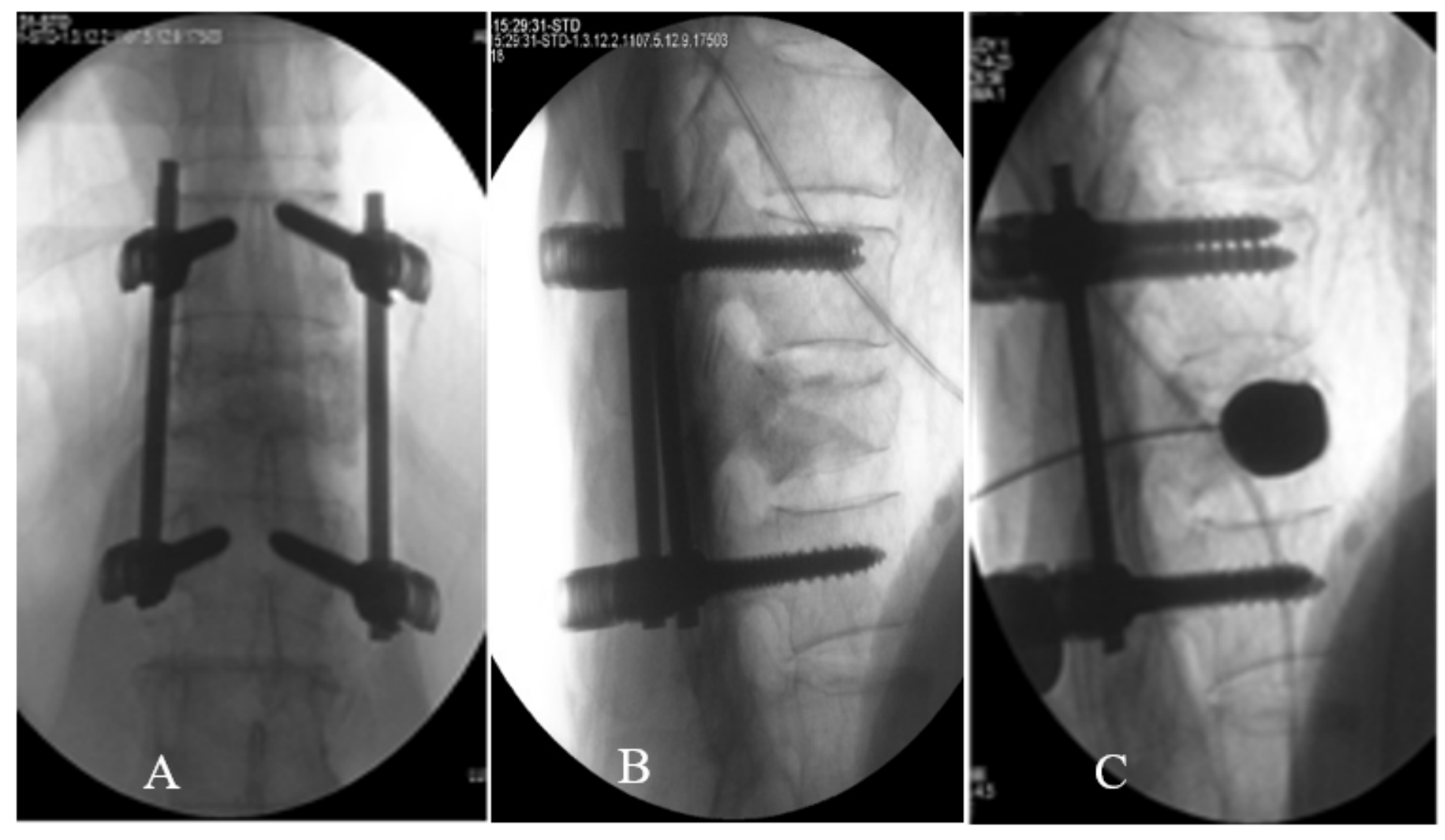

\section{Figure 4}

(A-F) Intraoperative C-arm fluoroscopy image picture $(A-F)$ Intraoperative C-arm fluoroscopy image picture shows that the height of L1 was partially recovered, kyphosis was slightly corrected, bone cement dispersion was good and no leakage occurred. Pedicle screw cutting vertebrae were noted in L2. 


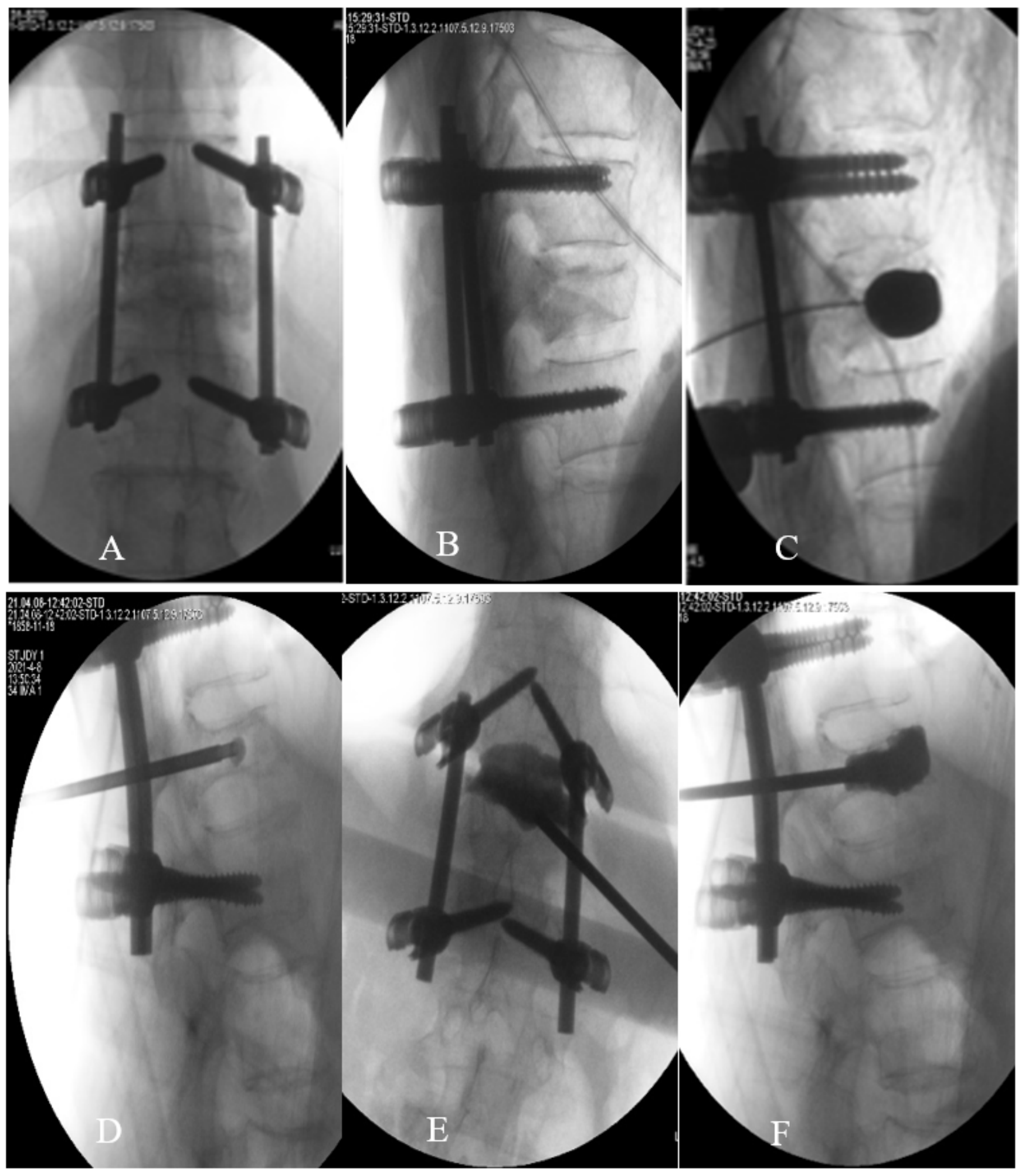

Figure 5

( $A$ and $B$ ) Postoperative positive and lateral $x$-ray. ( $A$ and $B$ ) Postoperative positive and lateral $x$-ray reveals the screw cutting vertebrae in L2. 